\title{
So früh lässt sich der Nutzen abschätzen
}

\begin{abstract}
Ob eine orale Eisensubstitution bei Patienten mit Eisenmangelanämie anschlägt, kann bereits nach zwei Wochen recht zuverlässig beurteilt werden.
\end{abstract}

In vielen Fällen lässt sich eine Eisenmangelanämie mit oralen Eisenpräparaten gut und günstig behandeln. Allerdings können vor allem gastrointestinale $\mathrm{Ne}$ benwirkungen die Adhärenz und den Therapieerfolg behindern.

Um den ausbleibenden Nutzen frühzeitig zu erkennen, empfehlen Ärzte vom Brigham and Women's Hospital in Boston, zwei Wochen nach Behandlungsbeginn erneut den Hämoglobin-Wert $(\mathrm{Hb})$ zu bestimmen. Wenn er zu diesem Zeitpunkt nicht um mindestens $1 \mathrm{~g} / \mathrm{d}$ l gegenüber dem Ausgangswert zugenommen hat, raten sie, auf Eisenpräparate zur I.v.Applikation zu wechseln.

\section{Analyse von Patienten mit oraler Eisensupplementation}

Ihre Empfehlung beruht auf einer Posthoc-Analyse von Patienten mit oraler Eisensupplementation aus fünf Studien, in denen ein Vergleich mit Eisen i.v. unter- nommen worden war. Die 738 Patienten, die meisten Frauen, hatten zu Beginn im Median einen $\mathrm{Hb}$ von 9,4 $\mathrm{g} / \mathrm{dl}$ sowie ein Serumeisen von $27 \mu \mathrm{g} / \mathrm{dl}$ und ein Serumferritin von 9,1 $\mathrm{ng} / \mathrm{ml}$. Bei der letzten Untersuchung nach 42 bzw. 56 Tagen war der Hb-Wert bei $89 \%$ der Patienten um mindestens $1 \mathrm{~g} / \mathrm{dl}$, bei $72 \%$ um mindestens $2 \mathrm{~g} / \mathrm{dl}$ und bei $43 \%$ um mindestens $3 \mathrm{~g} / \mathrm{dl}$ angestiegen.

\section{Positiver Vorhersagewert von 92,9\%} Ob letztlich ein Hb-Anstieg von wenigstens $2 \mathrm{~g} / \mathrm{dl}$ erreicht wurde, war relativ gut anhand des Ansprechens an Tag 14 abzulesen. 73\% der Patienten hatten zu diesem frühen Zeitpunkt beim $\mathrm{Hb}$ schon mindestens $1 \mathrm{~g} / \mathrm{dl}$ hinzugewonnen und wurden daher als Responder klassifiziert.

Ein Hb-Anstieg in dieser Größenordnung sagte mit einer Sensitivität von $90,1 \%$ und einer Spezifität von 70,3\% den längerfristigen Erfolg voraus. Der positive und der negative Vorhersagewert beliefen sich damit auf $92,9 \%$ und $72,7 \%$. Andere Parameter, die zu dieser Zeit erhoben wurden, erlaubten dagegen keinen
Rückschluss auf den Behandlungserfolg. Wie viele Patienten auf die Supplementation ansprachen, hing auch von der Ursache der Eisenmangelanämie ab. Am erfolgversprechendsten war die Behandlung bei einer postpartalen Anämie, 96\% der betroffenen Frauen waren Responder. Bei schweren uterinen Blutungen und gastrointestinalen Ursachen wurden Quoten von 56\% und 54\% erreicht, bei anderen Ursachen waren es $43 \%$.

Bei den Ergebnissen ist zu berücksichtigen, dass sie auf klinischen Studien beruhen. Die dort erzielte Therapieadhärenz von $84-99 \%$ dürfte deutlich höher als unter Alltagsbedingungen sein.

\section{Dr. Beate Schumacher}

Okam MM et al. Iron supplementation, response in iron-deficiency anemia: analysis of five trials. The American Journal of Medicine 2017 (online first)

\section{Hier steht eine Anzeige.}

